\title{
Estudios de imágenes en el diagnóstico precoz de leuce- mia en pediatría
}

\author{
Drs. Viviana Riquelme $\mathbf{S}^{(1)}$, Cristián García B(2).
}

1. Médico, egresada Facultad de Medicina Pontificia Universidad Católica. Santiago, Chile.

2. Departamentos de Radiología y Pediatría. Facultad de Medicina, Pontificia Universidad Católica. Santiago, Chile.

\section{Imaging studies in early diagnosis of childhood leukemia}

\begin{abstract}
Leukemia is the most commonly encountered cancer in children under the age of 15 and accounts for approximately 35-40\% of all cancers at that age. Acute lymphoblastic leukemia (ALL) is the most common type of leukemia affecting young children between the ages of 2 and 5 years; it accounts for around $80 \%$ of all childhood leukemia cases. Initial clinical and laboratory findings may be non-specific. Imaging studies in patients with bone pain and extramedullary involvement may provide the clinician with valuable supplementary information when the first symptoms result from tissue infiltration and haematological series show a discrete and asymptomatic involvement. The purpose of this work is to review the initial radiological manifestations of leukemia in children. Given the systemic nature of this disease, the goal is to identify the key elements found in the study of the different affected organs, in order to facilitate an early diagnosis of this condition. Late-stage alterations or treatment complications, such as osteonecrosis, infections by opportunistic pathogens, graft-versus-host disease, etc. -where magnetic resonance imaging (MRI) and computed tomography (CT) play a fundamental role- will not be reviewed.
\end{abstract}

Keywords: Child, Imaging, Leukemia, Pediatrics.

Resumen: La leucemia es el cáncer más frecuente en niños menores de 15 años y corresponde aproximadamente al 35-40\% de todos los cánceres a esa edad. Se presenta con mayor frecuencia entre los 2 y 5 años de edad y la forma más frecuente es la leucemia linfoblástica aguda, que corresponde al $80 \%$ de los casos. Las manifestaciones clínicas y de laboratorio pueden ser inicialmente inespecíficas. El estudio imagenológico en pacientes con dolor óseo y compromiso extramedular, puede entregar valiosa información complementaria al clínico cuando los primeros síntomas derivan de la infiltración de los tejidos y el compromiso de las series hematológicas es discreto y asintomático. El propósito de este trabajo es revisar las manifestaciones radiológicas iniciales de la leucemia en el niño. Considerando que es una enfermedad sistémica, el objetivo es identificar los elementos claves en el estudio de los distintos órganos comprometidos, que permitan al radiólogo sospechar el diagnóstico en la etapa precoz de la enfermedad. No se revisarán las alteraciones de la fase tardía o de complicaciones del tratamiento, como osteonecrosis, infecciones por gérmenes oportunistas, enfermedad de injerto vs. huésped, etc., donde la resonancia magnética (RM) y la tomografía computada (TC) juegan un rol fundamental.

Palabras clave: Imágenes, Niño, Leucemia, Pediatría.

Riquelme $V$ y cols. Estudios de imágenes en el diagnóstico precoz de leucemia en pediatría. Rev Chil Radiol 2012; 18(1): 24-29.

Correspondencia.Dr. Cristián García B. / cgarcia@med.puc.cl

Trabajo recibido el 24 de noviembre de 2011, aceptado para publicación el 19 de marzo de 2012.

\section{Introducción}

La incidencia esperada de cáncer en niños menores de 15 años, es de 110-150/1.000.000 niños por año, siendo algo más frecuente en varones ${ }^{(1)}$. La leucemia es el cáncer más frecuente y corresponde aproximadamente al $35-40 \%$ de todos los cánceres a esa edad(1).
Estadísticas norteamericanas establecen una incidencia de un caso de leucemia cada 7.000 niños/ año(2).

En Chile el informe preliminar del Registro $\mathrm{Na}$ cional Poblacional de Cáncer Infantil (RENCI) en el año 2007 reveló una incidencia de leucemias de $42.7 / 1.000 .000^{(1)}$. 
Desde el comienzo del Programa de Cáncer en el Niño en el año 1988, en el sector público se ha presentado un promedio de 100-110 casos nuevos de leucemia linfoblástica y 30 de leucemia mieloide por año ${ }^{(1)}$.

Los síntomas y signos que hacen sospechar una leucemia son variados y las recomendaciones para la sospecha diagnóstica están bien explicitadas en la Guía Clínica Leucemia en menores de 15 años, del Ministerio de Salud de Chile ${ }^{(1)}$.

En general, el diagnóstico se debe sospechar en todo niño con anemia no asociada a sangramiento, cuadro febril asociado o no con palidez, visceromegalia y hemograma con anemia y/o neutropenia (a veces leucocitosis), dolor óseo no asociado a traumatismo, con hemograma con citopenia.

La leucemia debe incluirse en el diagnóstico diferencial ante la presencia de los siguientes signos y síntomas combinados: Fiebre de origen desconocido; dolor óseo no asociado a traumatismos; hematomas espontáneos o asociados a traumas menores; sangramiento nasal o gingival; infecciones recurrentes; adenopatías (adenopatías persistentes por más de 4 semanas, ganglios mayores de $3 \mathrm{~cm}$, ganglios palpables en forma generalizada); y esplenomegalia ${ }^{(1-4)}$.

Existen factores genéticos y ambientales ligados a su patogenia. Es claro el aumento del riesgo asociado a genopatías como el Síndrome de Klinefelter y Síndrome de Down, presentando este último un riesgo relativo 15 veces superior a la población general ${ }^{(2,3)}$. La exposición a químicos como el benceno, pesticidas y radiación ionizante son factores modificables involucrados en su etiología(2).

La leucemia linfoblástica aguda (LLA) se define por la presencia de más de $20 \%$ de linfoblastos en médula ósea ${ }^{(2,3)}$ y es el tipo de leucemia más común durante la infancia, responsable del $80 \%$ de los $\operatorname{casos}^{(3,4)}$. Predomina en hombres y se presenta con mayor frecuencia entre los 2 y 5 años de edad ${ }^{(3,4)}$.

La leucemia mieloide aguda (LMA) representa el $17 \%$ de las leucemias ${ }^{(2)}$. Su presentación es igual en ambos sexos y no tiene predominio por rango etario ${ }^{(4)}$.

Las manifestaciones clínicas iniciales suelen ser inespecíficas y el cuadro de presentación más común incluye: palidez, fatiga, sangramientos, fiebre e infecciones recurrentes producto de la disfunción medular existente $^{(2,4)}$. En el hemograma, puede observarse anemia, trombopenia y/o neutropenia, con recuento leucocitario normal, disminuido o aumentado ${ }^{(3,4)}$. En el $95 \%$ de los casos, la LLA se manifiesta con alguna citopenia en el hemograma, con anemia en el $99 \%$ de los casos. El recuento leucocitario normal en $50 \%$ de los niños ${ }^{(3)}$ y en estos casos es la anemia el signo de alarma.

En la LMA el $44 \%$ de los pacientes tiene anemia al momento del diagnóstico, $69 \%$ neutropenia y el $25 \%$ tiene leucocitosis sobre $100.000 /$ microlitro $^{(3)}$, pudiendo presentar complicaciones como infartos cerebrales, pulmonares y hepáticos como consecuencia de la leucocitosis ${ }^{(1,3)}$.

Si bien el diagnóstico definitivo se obtiene mediante el estudio de la médula ósea, el estudio imagenológico, principalmente en pacientes con dolor óseo y compromiso extramedular, puede entregar valiosa información complementaria al clínico cuando los primeros síntomas derivan de la infiltración de los tejidos y el compromiso de las series hematológicas es discreto y asintomático.

La posibilidad de curación depende del tipo de leucemia, de la presencia de factores adversos asociados y de la respuesta al tratamiento, siendo alrededor de $73 \%$ para leucemia linfoblástica y $50 \%$ para leucemia mieloide ${ }^{(1)}$.

El propósito de este trabajo es revisar las manifestaciones radiológicas iniciales de la leucemia en el niño. Considerando que es una enfermedad sistémica, el objetivo es identificar los elementos claves en el estudio de los distintos órganos comprometidos, que permitan al radiólogo sospechar el diagnóstico en la etapa precoz de la enfermedad.

No se revisarán las alteraciones de la fase tardía o de complicaciones del tratamiento de la enfermedad, como osteonecrosis, infecciones por gérmenes oportunistas, enfermedad de injerto vs. huésped, etc., donde la resonancia magnética $(\mathrm{RM})$ y la tomografía computada (TC) juegan un rol fundamental.

\section{Leucemia. Manifestaciones óseas y extramedulares}

Debido a la infiltración de tejidos extramedulares, es posible observar visceromegalia (hepatomegalia, esplenomegalia, nefromegalia, aumento de tamaño del páncreas), poliadenopatías, compromiso de sistema nervioso central, renal y testicular ${ }^{(1,3,4)}$, siendo esta última la menos común. Su frecuencia es variable según el tipo de leucemia.

A continuación revisaremos las alteraciones radiológicas más frecuentes de los sitios u órganos que se comprometen con mayor frecuencia en esta enfermedad.

\section{Hígado, bazo, riñones}

El $60-70 \%$ de los pacientes con LLA debuta con hepatoesplenomegalia(3), mientras que en la LMA esto no ocurre en más de la mitad de los casos ${ }^{(5)}$. Suele ser un hallazgo al examen físico y rara vez ocasiona alteraciones funcionales ${ }^{(1)}$. La nefromegalia bilateral es también un hallazgo común, pero menos frecuente.

La ultrasonografía (US) permite demostrar el aumento de tamaño de estos órganos, lo que se puede asociar a disminución en la ecogenicidad del parénquima hepático y esplénico y aumento de ecogenicidad del parénquima renal, todo lo cual puede revertirse posterior al tratamiento ${ }^{(6,7)}$ (Figura 1). Además de nefromegalia, la tomografía compu- 
tada puede demostrar múltiples zonas de menor atenuación en el parénquima renal, generalmente bilateral $^{(7,8)}$ (Figura 2).

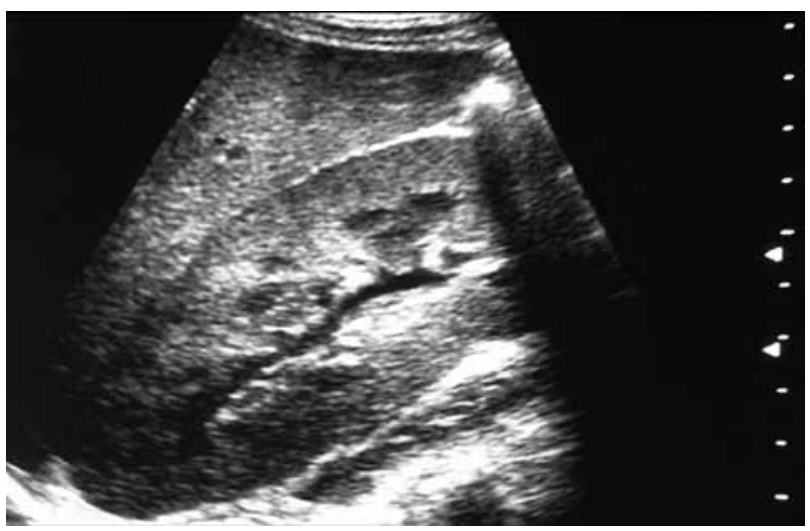

Figura 1. Niño de 6 meses. LLA. US abdominal mostró nefromegalia bilateral. En un corte sagital del flanco derecho se observa aumento de tamaño del riñón de ese lado (9,0 cm de diámetro sagital) y discreto aumento de su ecogenicidad.
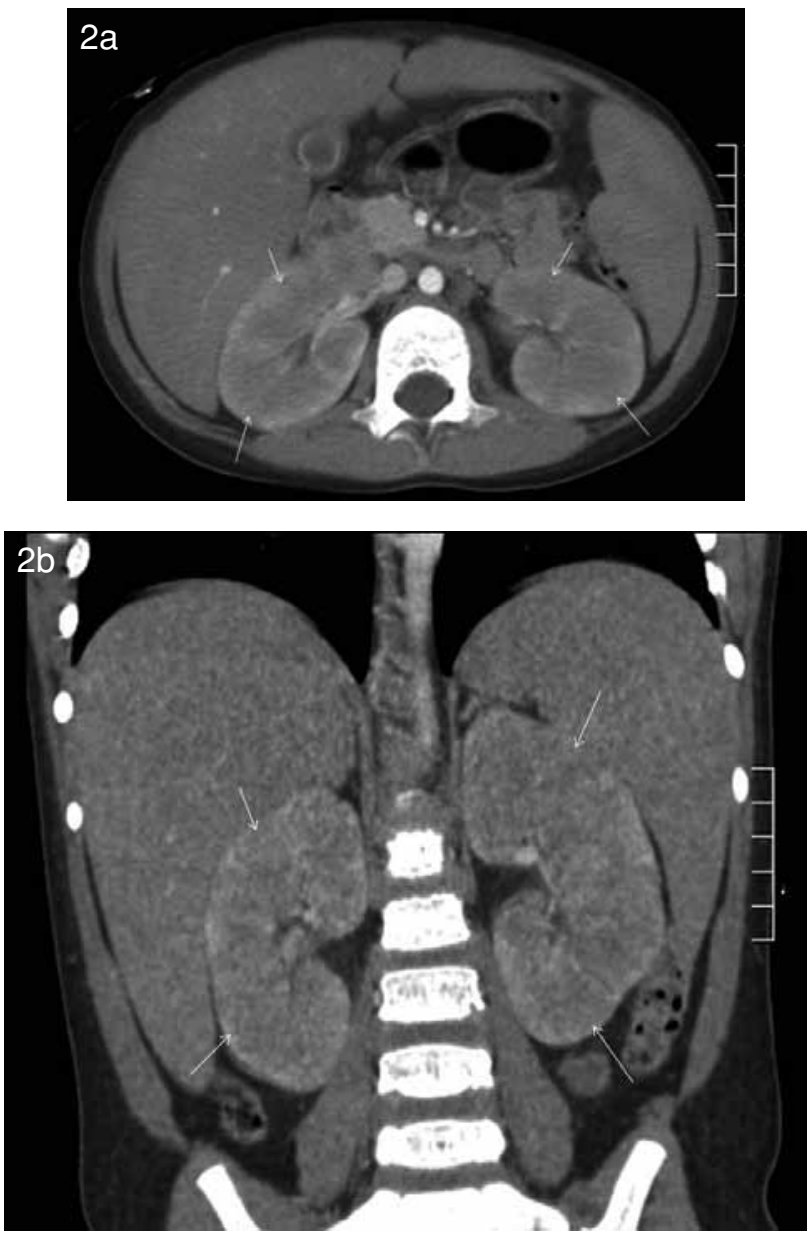

Figura 2. Niño de 4 años con LLA. TC de abdomen muestra en corte axial (a) y reconstrucción coronal (b), aumento de tamaño de ambos riñones, con múltiples áreas de menor atenuación en el parénquima (flechas). El niño presentaba además hepatoesplenomegalia.

\section{Linfoadenopatías}

El compromiso de los ganglios cervicales, axilares y/o inguinales ocurre hasta en el $50 \%$ de los pacientes en LLA y en el $25 \%$ de los pacientes con $\operatorname{LMA}^{(3,5)}$. La US es útil en el estudio de las adenopatías, donde además de confirmar su presencia, permite su caracterización ${ }^{(9,10)}$.

Generalmente, las adenopatías se asocian a hepatoesplenomegalia ${ }^{(9)}$, son indoloras, de rápido crecimiento, locales o generalizadas ${ }^{(10)}$. Se consideran sospechosos aquellos ganglios linfáticos de consistencia dura, de diámetro anteroposterior mayor de 1,0 $\mathrm{cm}$ en los ganglios axilares o cervicales y mayor de $1.5 \mathrm{~cm}$ en la región inguinal( ${ }^{(9)}$.

La morfología ganglionar oval normal puede estar conservada o ser de forma más redondeada y es común que se agrupen en racimo ${ }^{(9)}$. Las adenopatías son generalmente hipoecogénicas y el hilio ganglionar puede estar ausente o ubicarse excéntricamente producto de la infiltración tumoral ${ }^{(10,11)}$. En el estudio Doppler-color generalmente muestra flujo vascular de tipo hiliar en su interior. Si bien ninguno de los signos antes mencionados es patognomónico de leucemia, su presencia en el contexto clínico adecuado aumenta el índice de sospecha ${ }^{(11)}$.

\section{Compromiso óseo}

El dolor óseo es el síntoma más común, secundario a la proliferación de células neoplásicas en la médula ósea ${ }^{(7,12,13)}$.

El $30 \%$ de los pacientes con LLA se presenta con dolor óseo ${ }^{(4)}$ y en ocasiones, éste puede ser el único síntoma de alarma en presencia de un hemograma sin leucocitosis, lo que puede ocurrir en el $1 \%$ de los $\operatorname{casos}^{(3)}$. Puede comprometer cualquier segmento del esqueleto, pero las zonas más comúnmente afectadas son la pelvis, cuerpos vertebrales y huesos largos de extremidades inferiores ${ }^{(3)}$.

Clínicamente, el dolor suele ser de comienzo insidioso, constante, que no se relaciona con la actividad física y se exacerba durante la noche. Es de intensidad progresiva y refractario al uso de analgesia. Puede acompañarse de claudicación y rara vez se presenta inicialmente con fracturas en hueso patológico ${ }^{(4,13)}$.

Aunque es inusual, puede presentarse como oligoartralgias migratorias de grandes articulaciones, de distribución asimétrica, con o sin artritis. La similitud con enfermedades como artritis idiopática juvenil pueden retrasar el diagnóstico de leucemia ${ }^{(13)}$.

Pese a la amplia variedad de métodos de imágenes disponibles hoy en día, la radiografía $(\mathrm{Rx})$ simple continúa siendo el método inicial de elección para la evaluación del dolor óseo ${ }^{(14)}$. El 50-70\% de los pacientes con leucemia tienen alteraciones óseas evidentes en la radiografía al momento del diagnóstico ${ }^{(15,16)}$. 
Estas alteraciones consisten en:

1. Bandas radiolúcidas, finas, transversales, de ubicación metafisiaria. Son el hallazgo más común y sugerente de leucemia a partir de la edad preescolar. En pacientes menores de 2 años, su especificidad disminuye y pueden observarse en otras enfermedades crónicas que también alteren la osteogénesis ${ }^{(6,12,15)}$ Suelen localizarse en húmero proximal, radio distal y fémur distal ${ }^{(7,14)}$, siendo este último el sitio más frecuente ${ }^{(7,13)}$ (Figuras 3 y 4 ).

2. Osteopenia local o difusa, lo que puede manifestarse con dolor dorsal o lumbar por aplastamiento vertebral ${ }^{(6,7,15)}$ (Figura 5).

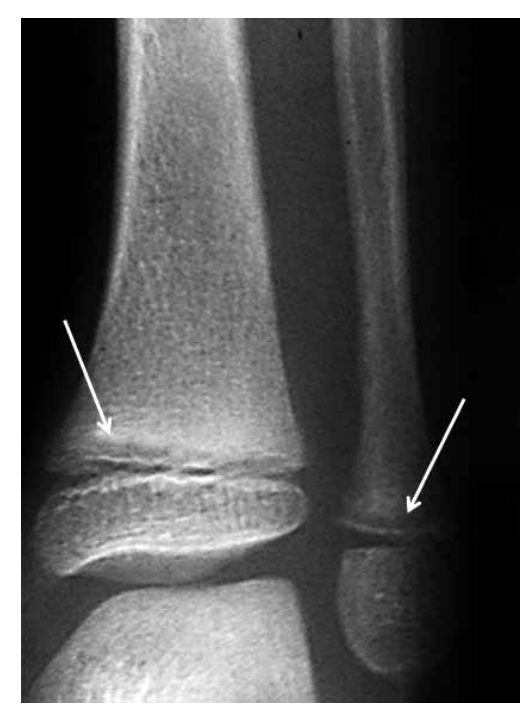

Figura 3. Niño de 5 años. Compromiso óseo en leucemia. $R x$ anteroposterior del tobillo izquierdo muestra bandas radiolúcidas bien definidas en las metáfisis distales de la tibia y el peroné (flechas).

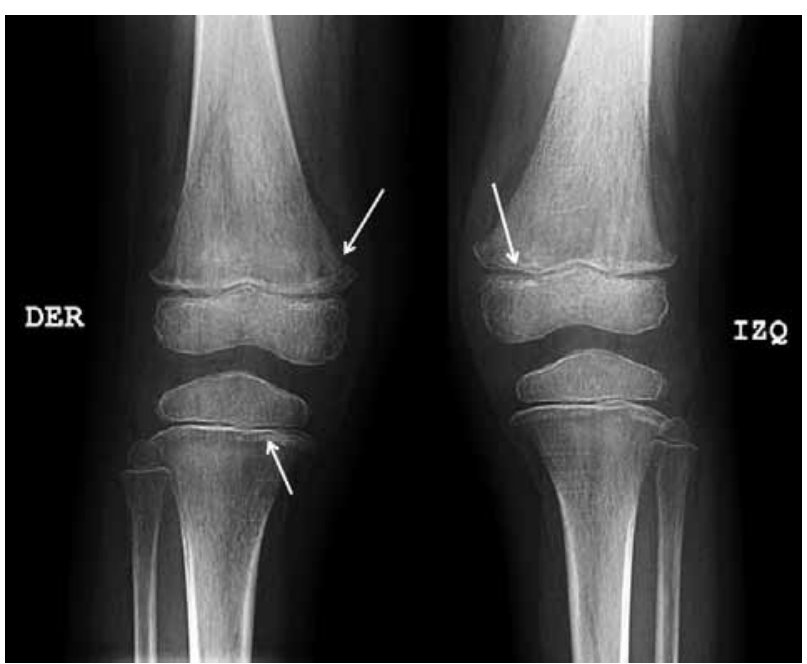

Figura 4. Compromiso óseo por leucemia. Rx anteroposterior de ambas rodillas muestra osteopenia difusa y bandas radiolúcidas metafisiarias relativamente mal definidas en ambos fémures y tibias (flechas).

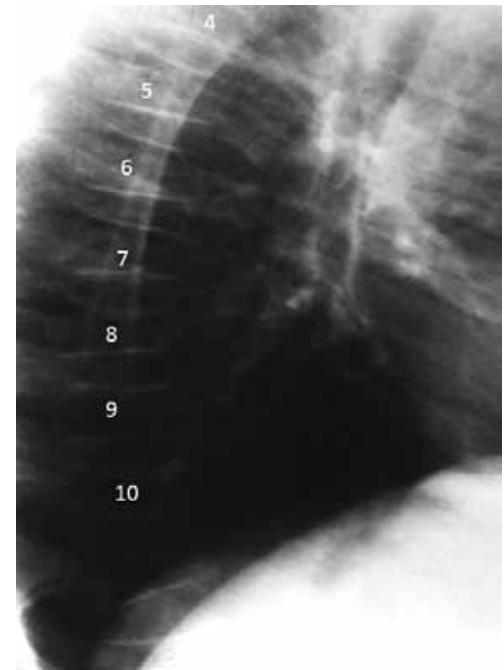

Figura 5. Niño 12 años. Compromiso óseo por leucemia. $R x$ lateral de la columna dorsal, muestra osteopenia difusa y aplastamiento de todos los cuerpos vertebrales dorsales visibles (D4-D10).

3. Osteolisis focal o generalizada de huesos largos y planos (cráneo, pelvis, costillas y cintura escapular) ${ }^{(14)}$. Puede presentar aspecto de "hueso apolillado" o rarefacción ósea, por la presencia de pequeñas áreas líticas, de bordes mal definidos ${ }^{(17)}$.

4. Reacción perióstica y subperióstica, que corresponden a zonas de engrosamiento del periostio debido a neoformación ósea ${ }^{(6,7,15)}$. (Figura 6).

5. Osteoesclerosis, que es una manifestación tardía y poco frecuente(6).

6. Edema de tejidos blandos, osteoporosis yuxta-articular y derrame articular ${ }^{(15)}$.

Las bandas metafisiarias y lesiones osteolíticas pueden observarse hasta en el $70 \%$ de los casos, mientras que la osteopenia se ve hasta en $25 \%$ de los pacientes y las fracturas sólo en $10 \%$ de los niños ${ }^{(16)}$.

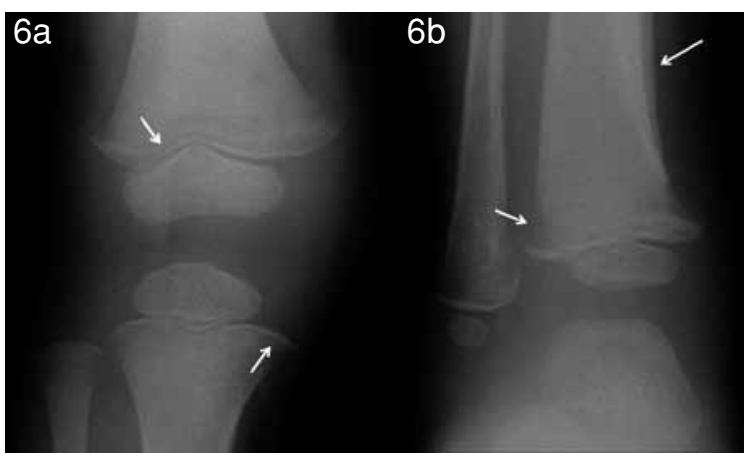

Figura 6. Compromiso óseo en leucemia en un niño de 8 años. (a) $R x$ anteroposterior de rodilla derecha (a) muestra bandas radiolúcidas metafisiarias en fémur y tibia (flechas). (b) $R x$ anteroposterior de tobillo derecho muestra zona osteolítica en el borde lateral de la metáfisis distal de la tibia y reacción perióstica de la diáfisis distal en su borde medial (flechas). 


\section{Mediastino}

El compromiso del mediastino ocurre típicamente en la LLA(1). El $15 \%$ de estos pacientes debuta con un cuadro clínico tipo linfoma, de los cuales el $61 \%$ presenta adenopatías mediastínicas ${ }^{(18,19)}$. La clínica es variable, puede manifestarse con tos, estridor, ortopnea, disnea, síndrome de vena cava superior o síndrome de distress respiratorio ${ }^{(1,3,4)}$.

Radiológicamente, corresponde a una masa del mediastino anterior, que se puede sospechar en la Rx simple y se confirma con tomografía computada (TC) de tórax (Figura 7). El diagnóstico diferencial incluye fundamentalmente linfoma.

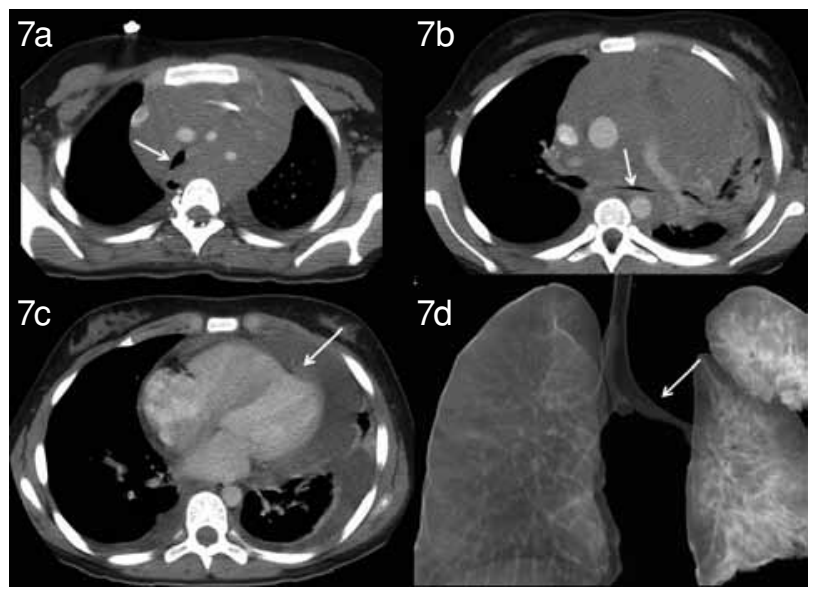

Figura 7. Niña de 13 años con LLA, que se presenta con masa mediastínica y estridor. TC de tórax muestra gran masa del mediastino anterior, que desplaza las estructuras vasculares y muestra (a) compresión y desplazamiento de la tráquea (flecha), (b) marcado estrechamiento del bronquio fuente izquierdo (flecha), (c) derrame pericárdico (flecha). En una reconstrucción $3 D$ de la vía aérea (d) se destaca la compresión del bronquio fuente izquierdo (flecha).

\section{Región mamaria}

El compromiso mamario por leucemia se manifiesta como un nódulo o masa mamaria, con mayor frecuencia unilateral, que puede estar presente al inicio de la enfermedad o durante su evolución. Al examen clínico, suelen ser tumores bien delimitados, de rápido crecimiento y en ocasiones, dolorosos ${ }^{(20)}$. En la US aparecen como nódulos hipoecogénicos, de márgenes bien o mal definidos, generalmente únicos ${ }^{(18,19)}$. Es poco frecuente que sean lesiones múltiples y bilaterales. Además, puede observarse edema difuso del tejido adyacente y adenopatías axilares $(20,21)$.

\section{Testículos}

La infiltración de los testículos ocurre principalmente en pacientes menores de 20 años ${ }^{(21)}$. Es poco frecuente que la leucemia comprometa este órgano en forma primaria, más bien, éste suele ser un sitio de recurrencia.
El cuadro clínico es el de un tumor testicular con dolor, sensación de pesadez y/o aumento de volumen escrotal que puede ser uni o bilateral(22,23).

En la US puede observarse aumento del tamaño testicular determinado por compromiso focal (con múltiples nódulos) o difuso, con ecogenicidad aumentada o disminuida, siendo más frecuente el compromiso difuso(23,24) (Figura 8). En algunos casos de LLA, también existe infiltración del epidídimo. En el estudio Doppler-color, hay un aumento del flujo sanguíneo(24).

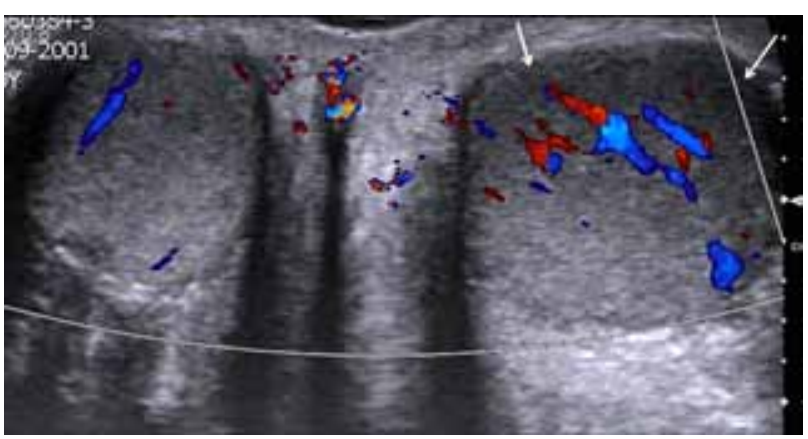

Figura 8. Niño de 9 años. LLA tratada y en remisión. Aumento de volumen testicular por recaída testicular. US Doppler color escrotal en un corte trasversal, muestra aumento de tamaño del testículo izquierdo, con un área de menor ecogenicidad y flujo vascular aumentado en su mitad anterior (flechas).

\section{Conclusiones}

- Los síntomas y signos que hacen sospechar una leucemia aguda en el niño son variados y las manifestaciones clínicas iniciales suelen ser inespecíficas.

- El estudio imagenológico puede entregar valiosa información al clínico, cuando los primeros síntomas derivan de la infiltración de los tejidos y el compromiso de las series hematológicas es discreto y asintomático.

- Por este motivo el médico radiólogo debe conocer las manifestaciones radiológicas iniciales de la enfermedad en el niño, en especial el compromiso extramedular y así sospechar el diagnóstico en su etapa precoz.

- En especial, el radiólogo debe considerar la leucemia dentro del diagnóstico diferencial en niños con dolor óseo, visceromegalia y masas del mediastino anterior.

\section{Bibliografía}

1. Ministerio de Salud de Chile: Guía Clínica Leucemia en menores de 15 años. Actualizado 2010. Disponible en: http://www.minsal.gob.cl/portal/url/item/7220fd c433e944a9e04001011f0113b9.pdf Consultado 25 Septiembre 2011.

2. Hutter J. Childhood Leukemia. Pediatr Rev. 2010; 31: 234-241.

3. Maloney K, Foreman N, Giller R, Quinones R et al : Neoplastic Disease. Hay W, Jr., En: Levin M, Sondheimer J, Deterding R: Current Diagnosis and Treatment: 
Pediatrics. 20th Edition, 2011. Chapter 29. Disponible en: http://www.accessmedicine.com/resourceTOC. aspx?resourcelD $=14$.

4. Pearce J, Sills R. Consultation with the Specialist: Childhood Leukemia. Pediatr Rev 2005; 26; 96-104.

5. Aquino V. Acute myelogenous leukemia. Current Problems in Pediatric and Adolescent Health Care 2002; 32: 50-8.

6. Rosenfield N: Part I. Early findings. En: The Radiology of Childhood Leukemia and its therapy. Second edition. Warren H. Green, INC. St Louis, U.S.A, 1982; 5-19.

7. Guillerman RP, Voss SD, Parker BR. Leukemia and Lymphoma. Radiol Clin N Am 2011; 49: 767-797.

8. Hilmes MA, Dillman JR, Mody RJ, Strouse PJ.Pediatric renal leukemia: spectrum of CT imaging findings. Pediatric Radiology 2007; 37: 896-907.

9. Friedmann AM. Evaluation and management of lymphadenopathy in children. Pediatr Rev 2008; 29: 53-60.

10. Restrepo R, Oneto J, Lopez K, Kukreja K. Head and neck lymph nodes in children: the spectrum from normal to abnormal. Pediatr Radiol 2009; 39: 836-46.

11. Ahuja AT, Ying M. Sonographic evaluation of cervical lymph nodes. Am J Roentgenol 2005; 184: 1691-1699.

12. Vargas L, Miranda M. Manifestaciones osteoarticulares en la presentación inicial de la leucemia linfoblástica aguda del niño. Rev Chil. Pediatr 1995; 66: 98-102.

13. Serra-Bonett N, Guzmán Y, Rodríguez E, Millana A, Rodríguez M. Leucemia aguda en niños con diagnóstico erróneo de artritis idiopática juvenil. Reumatol Clin 2008; 4: 70-73.

14. Wyers M. Evaluation of pediatric bone lesions. Pediatr
Radiol 2010; 40:468-473.

15. Resnick D, Haguigui P. Enfermedades Mieloproliferativas. En: Resnick D. Huesos y articulaciones en imagen. Madrid: Editorial Marbán 2001; 625-627.

16. Davies JH, Evans BA, Jenney ME, Gregory JW. Skeletal morbidity in childhood with acute lymphoblastic leukaemia. Clin Endocrinol 2005; 63: 1-9.

17. Miller T. Radiography of Bone Tumors and Tumor-like Conditions: analysis with conventional radiography. Radiology 2008; 246: 662-674.

18. Merten D. Diagnostic imaging of mediastinal masses in children. Am J Roentgenol 1992; 158: 825-832.

19. Meyer JS, Nicotra JJ. Tumors of the pediatric chest. Semin Roentgenol 1998; 33: 187-198.

20. Chung EM, Cube R, Hall GJ, González C, et al. From the archives of the AFIP: breast masses in children and adolescents: radiologic-pathologic correlation. Radiographics 2009; 29: 907-931.

21. García CJ, Espinoza A, Dinamarca V, Navarro O, et al. Breast US in children and adolescents. Radiographics 2000; 20: 1605-1612.

22. Kundra V. Testicular cancer. Seminars in Roentgenology 2004; 39: 437-450.

23. Woodward PJ, Sohaey R, O' Donoghue MJ, Green DE. From the archives of the AFIP: tumors and tumor-like lesions of the testis: radiologic-pathologic correlation. Radiographics 2002; 22: 189-216.

24. Aso C, Enríquez G, Fité M, Torán N, et al. Gray-scale and color Doppler sonography of scrotal disorders in children: an update. Radiographics 2005; 25: 1197-1214. 